\title{
Ex vivo modelling of drug efficacy in a rare metastatic urachal carcinoma
}

Rami Mäkelä ${ }^{1}$, Antti Arjonen ${ }^{1,2}$, Ville Härmä1,3, Nina Rintanen ${ }^{4}$, Lauri Paasonen ${ }^{5}$, Tobias Paprotka ${ }^{6}$, Kerstin Rönsch , Teijo Kuopio ${ }^{4}$, Juha Kononen ${ }^{4,7}$ and Juha K. Rantala ${ }^{1,3^{*}}$ (D

\begin{abstract}
Background: Ex vivo drug screening refers to the out-of-body assessment of drug efficacy in patient derived vital tumor cells. The purpose of these methods is to enable functional testing of patient specific efficacy of anti-cancer therapeutics and personalized treatment strategies. Such approaches could prove powerful especially in context of rare cancers for which demonstration of novel therapies is difficult due to the low numbers of patients. Here, we report comparison of different ex vivo drug screening methods in a metastatic urachal adenocarcinoma, a rare and aggressive non-urothelial bladder malignancy that arises from the remnant embryologic urachus in adults.
\end{abstract}

Methods: To compare the feasibility and results obtained with alternative ex vivo drug screening techniques, we used three different approaches; enzymatic cell viability assay of 2D cell cultures and image-based cytometry of 2D and 3D cell cultures in parallel. Vital tumor cells isolated from a biopsy obtained in context of a surgical debulking procedure were used for screening of 1160 drugs with the aim to evaluate patterns of efficacy in the urachal cancer cells.

Results: Dose response data from the enzymatic cell viability assay and the image-based assay of 2D cell cultures showed the best consistency. With 3D cell culture conditions, the proliferation rate of the tumor cells was slower and potency of several drugs was reduced even following growth rate normalization of the responses. MEK, mTOR, and MET inhibitors were identified as the most cytotoxic targeted drugs. Secondary validation analyses confirmed the efficacy of these drugs also with the new human urachal adenocarcinoma cell line (MISB18) established from the patient's tumor.

Conclusions: All the tested ex vivo drug screening methods captured the patient's tumor cells' sensitivity to drugs that could be associated with the oncogenic KRAS ${ }^{G 12 V}$ mutation found in the patient's tumor cells. Specific drug classes however resulted in differential dose response profiles dependent on the used cell culture method indicating that the choice of assay could bias results from ex vivo drug screening assays for selected drug classes.

Keywords: Ex vivo drug screening, Urachal carcinoma, Rare cancer, Precision medicine

\footnotetext{
*Correspondence: rantala@misvik.com

${ }^{1}$ Misvik Biology Ltd, Karjakatu 35 B, Fl-20520 Turku, Finland

${ }^{3}$ University of Sheffield, Sheffield, UK

Full list of author information is available at the end of the article
}

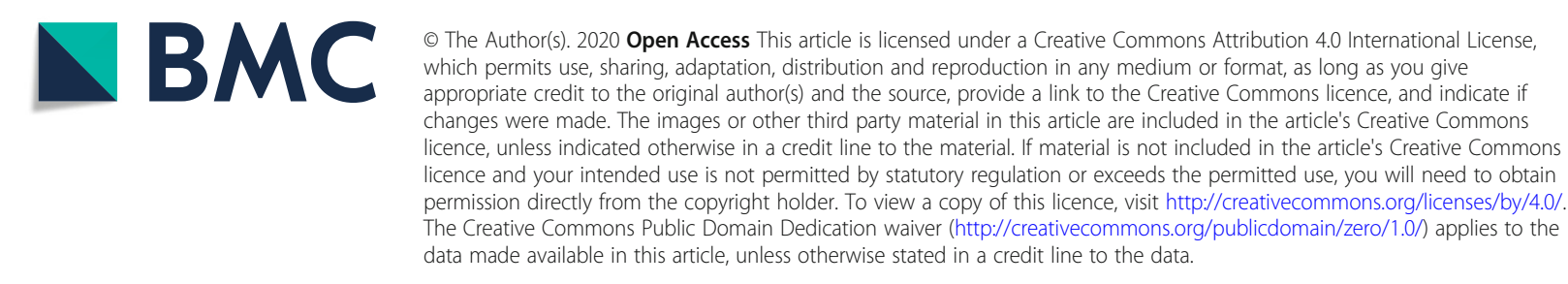




\section{Background}

The development of high-throughput screening technologies and cell culture methods has made it feasible to perform large-scale in vitro drug screens also using patient derived primary tumor cell cultures [1-3]. These techniques are collectively called as ex vivo drug screening methods. The utility of ex vivo drug screening has emerged as a novel approach to complement pathological cancer diagnostic procedures to track patient specific drug sensitivity to hundreds of cancer therapeutics in a single experiment [3]. The results can be used to confirm drug sensitivity patterns predicted from molecular genetics [2] or to inform treatment decision and personalized care of individual cancer patients when standard treatment options have been exhausted [3]. In context of rare cancers, the low number of patients limits the clinical evaluation and validation of novel treatment strategies using conventional trial mechanisms. Thus, demonstration of the efficacy of novel therapeutics in rare cancer types through empirical evidence from ex vivo tests or similar alternative models may be the only option to motivate clinical development of these treatments [3-5]. One such rare cancer, for which ex vivo evidence could be used as motivation for development of novel treatment strategies, is urachal cancer, an aggressive non-urothelial bladder malignancy accounting for less than $1 \%$ of all bladder cancers [6]. Urachal adenocarcinoma (UrAC) arises in adults from the vestigial musculofibrous remnant band that connects the allantois and the bladder during embryonic development. A large proportion of patients with UrAC initially present with an advanced disease [7] and patients with metastatic urachal cancer have a poor prognosis [8-11]. Given the rarity of urachal cancers, prospective trials to guide the treatment of patients with advanced disease are lacking, there are no standard chemotherapeutic regimens, and surgery remains the mainstay of therapy shown to improve the overall survival outcome of UrAC [12]. To date, no randomized trials of urachal carcinomas have been reported and the most comprehensive reviews to date have concluded 420 [10] and 456 [11] patients reported regionally and 1010 patients reported globally [13]. As a result, limited information exists regarding the effective management of these cancers beyond the use of chemotherapy including 5-fluorouracil based, 5-fluorouracil and cisplatin [14-17] or hyperthermic intraperitoneal chemotherapy [18-20]. Especially, knowledge concerning the efficacy of new genome aberration targeted chemotherapeutic agents is limited to a handful of case reports from individual institutions [14, 21-25]. Moreover, comprehensive tumor genomic profiling of UrAC samples has not been described and the only common genetic features described in the limited number of reported cases have included aberration of
APC, BRAF, EGFR, KRAS, PIK3CA, TP53 and microsatellite instability [21-26]. To improve our understanding of the disease pathogenesis and therapy sensitivity of UrAC, we performed a large-scale ex vivo drug screening of 1160 drugs with vital tumor cells derived from a patient with a metastatic urachal adenocarcinoma. In this study, we also compared the reproducibility of results derived with three different high-throughput drug screening approaches to assess assay dependency of the ex vivo measured dose responses of the patient derived tumor cell cultures. Last we describe establishment of a new human urachal adenocarcinoma cell line (MISB18) which is the first described UrAC cell line with a known genetic background.

\section{Methods}

Patient derived primary tumor cell culture

The patient was identified to the study by an oncologist at Jyväskylä Medical Central, Finland. A subcutaneous metastasis tissue sample was collected for the study during palliative surgery. In conjunction with the surgical procedure, part of the dissected tumor tissue was placed in sterile RMPI-1640 medium (Gibco) for transport to the consulting pathologist for preparation and further delivery to the research laboratory (Fig. 1a). Rest of the tissue was fixed in $4 \%$ buffered formaldehyde, paraffinembedded, cut at $4 \mu \mathrm{m}$, and subjected to routine staining procedures including hematoxylin and eosin stain (H\&E) and pathological evaluation (Fig. 1b). The live tissue was dissociated into a single cell suspension as described before [3]. Following the enzymatic dissociation, the resulting cell suspension was counted using a Cellometer Mini cell counter (Nexcelom). In total $6.5 \times 10^{\wedge} 6$ cells with an average size of $>13 \mu \mathrm{m}$ was derived from $\sim 2 \mathrm{~cm}^{3}$ of the tumor tissue. The suspension was diluted to RPMI-1640 medium (Gibco) containing 5\% FBS to achieve a suspension with 1000 cells per $45 \mu \mathrm{L}$ of medium. $5 \times 10^{\wedge} 6$ cells were used for the initial ex vivo drug screening and the rest were placed to cell culture in standard cell culture conditions $\left(37^{\circ} \mathrm{C}, 5 \% \mathrm{CO}_{2}\right)$. Following 4 days in culture, the cells presented a semiadherent phenotype with cells growing both as loose aggregates and adhered to the plastic cell culture surface (Fig. 1b). The use and investigation of the patient derived cells was approved by the local Ethics Committee of the Central Finland Health Care District (KSSHP 3 U/ 2015). All the experiments were undertaken with the understanding and written informed consent of the patient. The study methodologies conformed to the standards set by the Declaration of Helsinki.

\section{Ex vivo drug screening}

The therapeutic compound collection used in the ex vivo study consisted of 1140 FDA approved drugs, 


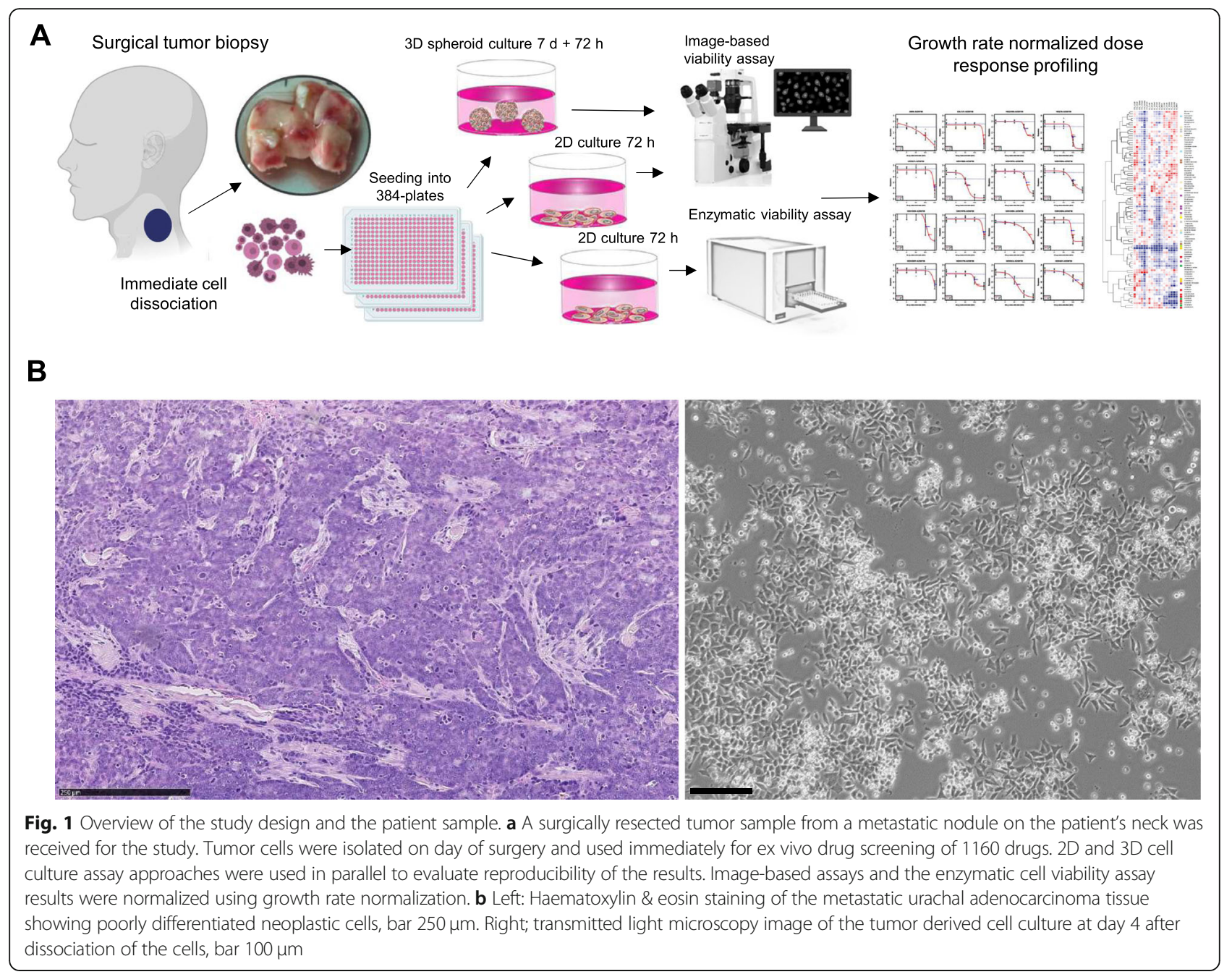

purchased as single a collection of FDA approved drugs from a commercial chemical vendor (Cat.no. L1300, Selleck biochemicals, Houston, TX, USA) readily dissolved in dimethyl sulfoxide (DMSO). The library was supplement with 20 investigational and preclinical compounds covering key cancer associated signaling pathway targets including AKT, ATR, BET (bromodomain), EGFR, FGFR, MDM2, MEK, PIM, PI3K, pan-RAF and WEE1. Since platinum-based drugs (cisplatin, carboplatin and oxaliplatin) are inactivated by DMSO, these were replaced in the compound library with stock solutions diluted in physiological saline. The ex vivo drug screening experiments were performed in 384-well microplate format as described before [3]. Briefly, each compound was tested in the initial high content image-based screening with three different concentrations in 2-fold dilutions starting from $5 \mu \mathrm{M}$ as the highest concentration. In the secondary screening experiments, the compounds were tested in five 2-fold concentrations starting from $5 \mu \mathrm{M}$ as the highest concentration. For the screening experiments performed with 2D cell cultures, the compounds were pre-printed on tissue culture treated 384-well plates (Corning, ThermoFisher Scientific) diluted in $5 \mu \mathrm{l}$ of RPMI-1640 medium without supplements with a liquid handling device (Eppendorf EpMotion-96, Eppendorf GmbH.). For the screening experiment performed with 3D cell cultures, the compounds were aliquoted on top of the $3 \mathrm{D}$ cell cultures in $10 \times$ concentration. Cell suspension of freshly isolated urachal carcinoma cells $(45 \mu \mathrm{l}$ per well; 1000 cells per well) was then transferred to each well using Multi-Drop Micro peristaltic dispenser (ThermoScientific). The 384-well plates were then incubated for $72 \mathrm{~h}$ at $37^{\circ} \mathrm{C}$ and $5 \% \mathrm{CO}_{2}$.

\section{Enzymatic cell viability assay}

To assess drug induced growth inhibition with an enzymatic cell viability assay following a 72-h incubation of the cells with drugs, cell viability was measured using CellTiter-Glo reagent (Promega) according to the manufacturer's instructions with Labrox luminescence plate reader (Labrox). Briefly, $20 \mu \mathrm{L}$ of the reagent was added 
per 384-well and incubated for $30 \mathrm{~min}$ at room temperature in gentle shaking. Cell viability luminescence data was normalized to median luminescence signal from $0.05 \%$ DMSO only wells (negative controls), 2 $\mathrm{mM}$ hydroxyurea containing wells (proliferation growth controls) and $5 \mu \mathrm{M}$ staurosporin containing wells (positive controls). Dose response was presented as growth rate (GR) normalized \% of signal in comparison to negative and positive control samples (see Statistical analysis).

\section{Image-based cell viability assays}

Microscopic image-based drug screening with 2D cell cultures was performed using an Olympus $\operatorname{scan}^{\wedge} \mathrm{R}$ integrated high content imager and image analysis suite (Olympus) equipped with a Hamamatsu ORCA-R2 CCD digital camera (Hamamatsu Photonics K.K). Each well was imaged individually with a $10 \times$ objective using specific filter sets for DAPI (Semrock). The scan ${ }^{\wedge} \mathrm{R}$ image analysis software suite was used for quantitative analysis of image features. Analysis capabilities included cell segmentation based on nuclear DNA staining and cell counting. The effects of each drug on cell counts as indicator of cell growth inhibition or cytotoxicity were assessed by comparing cell counts with comparable cell counts measured in DMSO only wells (negative controls) and $2 \mathrm{mM}$ hydroxyurea containing wells (proliferation controls). The DNA counterstaining of the cells was performed according to the following protocol. First the culture medium was aspirated carefully from each well and the cells were fixed with $2 \%$ paraformaldehyde (Sigma-Aldrich) in PBS for $15 \mathrm{~min}$ at room temperature. Cells were then washed once for $5 \mathrm{~min}$ with PBS. Cells were permeabilized with $0.3 \%$ Triton-X100 in $20 \mu \mathrm{L}$ of PBS for $15 \mathrm{~min}$ at room temperature, followed with a PBS wash. DAPI (4',6-diamidino-2-phenylindole, Invitrogen) DNA counterstaining was performed for $1 \mathrm{~h}$ at room temperature, followed by washing with PBS (Supplementary Figure 1).

\section{D cell culture assays with imaging cytometry}

The 3D cell culture assays were performed using GrowDex $^{\oplus}$ hydrogel (UPM, Helsinki, Finland) as the matrix supporting 3-dimensional cell growth. To minimize adherent cell growth in the bottom surface of the wells, the microwells were first coated with 1.2\% pHEMA (poly (2-hydroxyethyl methacrylate; Polysciences). For the experiment the $1.5 \%$ hydrogel stock was mixed with complete cell culture medium (RPMI-1640 + 5\% FBS) to achieve a $0.6 \% \mathrm{w} / \mathrm{v}$ hydrogel solution. $30 \mu \mathrm{L}$ of cell suspension containing 2000 cells was mixed with $30 \mu \mathrm{L}$ of the diluted hydrogel to achieve a $0.3 \% \mathrm{w} / \mathrm{v}$ hydrogel solution containing $\sim 30$ cells $/ \mu \mathrm{L}$. Required amount of hydrogel-cell solution was prepared by pipetting the hydrogel using a wide-mouth $10 \mathrm{~mL}$ pipetting tip into a $50 \mathrm{~mL}$ tube and gentle mixing with a vortex shaker. The hydrogel-cell solution was dispensed to 384-plate wells using a Multidrop plate dispenser (ThermoFisher Scientific). The total volume of sample added into a single 384-well was 60uL/well (2000 cells). Following dispensing, plates were centrifuged for $1 \mathrm{~min}$ at $100 \mathrm{~g}$ and left on incubation at $+37^{\circ} \mathrm{C}, 5 \% \mathrm{CO}_{2}$ for 7 days prior to addition of the drugs. At day 4, $20 \mu \mathrm{L}$ of medium was carefully aspirated from the wells using a multichannel pipet and $20 \mu \mathrm{L}$ of fresh complete cell culture medium was added to each well. On day 7 , the same was repeated followed by addition of $6 \mu \mathrm{L}$ of the $10 \times$ drug stocks per well and additional 72-h incubation. For analysis of cell growth, the cells were stained using Hoechst 33342 cell permeant live cell DNA dye (Invitrogen). $8 \mu \mathrm{L}$ of $10 \times$ stock dilution was added per well and incubated for 45 min. Following Hoechst staining, the plates were centrifuged for $2 \mathrm{~min}$ at $200 \mathrm{~g}$ to settle the cell spheroids/aggregates to the bottom plane of the wells (Supplementary Figure 1). Imaging and image analysis were then performed using an Olympus scan ${ }^{\wedge} \mathrm{R}$ high content imager and image analysis suite as described above for the $2 \mathrm{D}$ assays.

\section{Mutation analysis}

Targeted genomic profiling of an oncopanel with 850 cancer associated genes was performed from the ex vivo tumor cell culture following 1 month in culture. Genomic DNA was extracted from $1 \times 10^{\wedge} 6$ cells using NucleoSpin Tissue (Macherey-Nagel GmbH) DNA purification kit according to the manufacturer's protocol. Hybridization-based target capture was performed with Agilent SureSelect (Agilent) technology and sequencing libraries were sequenced using paired end $100 \mathrm{bp}$ read format on an Illumina HiSeq2500 instrument per the manufacturer's instructions (Illumina). Single nucleotide variants (SNVs), insertions and deletions (In/Del) were detected and filtered based on mutation allele frequency (>1\%). Detected variants were screened for known clinical significance in ClinVar (released 02. Oct 2017) database [27]. Result are available online at Mendeley data; DOI: https://doi.org/10.17632/kc7wmn3rcs.2.

\section{Statistical analysis}

Statistical analysis was performed with the Microsoft Excel, Cluster 3.0 and GraphPad Prism 7 statistical software. The ex vivo drug screening data was analyzed using the normalized growth rate inhibition (GR) approach which yields perdivision metrics for drug potency and efficacy. The normalized growth rate inhibition (GR) method corrects for variation in division rates by estimating the magnitude of drug response on a per cell-division basis. The GR values were used for comparison of drug potency between the 
different screening methods to correct for differential proliferation rate of the cells in 2D and 3D culture conditions. GR values were calculated as previously described [2,3]. Combination indices $(\mathrm{CI})$ were calculated from replicate, fixedratio, dose escalation experiments using the Chou and Talalay method [28]. CI values were reported at 50\% inhibitory values (CI50). IC50 values were calculated with GraphPad Prism 7 software using a nonlinear curve fit equation. Due to the limited number of primary cells available for technical replicate screening experiments, different drug test doses were considered as biological replicates, and the corresponding $p$-values were calculated across the dilution series with Welch's t-test according to assumptions on data normality.

\section{Results}

\section{Ex vivo drug screening of urachal cancer cells}

The patient, a 36-year-old male was diagnosed with a 9 $\mathrm{cm}$ cystic-solid tumor in front of bladder. The tumor cells infiltrating bladder epithelium demarcated sharply from the urothelium. Initial treatment included cystectomy and wide pelvic lymphadenectomy. Pathological evaluation of the surgical preparation confirmed the diagnosis of urachal mucinous cystadenoma and poorly differentiated urachal carcinoma. Carcinoma fraction of the tumor contained various different regions with mixed histological features, including partial differentiation to urothelial carcinoma, intestinal carcinoma and squamous cell carcinoma. Cancer showed high proliferation rate, mitotic figure count was 40/10 HPF. Up to $30 \%$ of the tumor contained necrotic tissue. Adjuvant treatment of the patient consisted of six cycles of cisplatin - 5-FU regimen. Immediately after adjuvant chemotherapy patient presented with subcutaneous metastatic lesion located behind left ear. Patient received radiotherapy with a palliative intent ( $30 \mathrm{~Gy}, 10 \times 3 \mathrm{~Gy}$ ). Disease progressed shortly after completing radiotherapy course and second-line systemic chemotherapy was initiated with docetaxel - gemcitabine. Disease progressed again and new lesions appeared in multiple locations (neck, axilla, sternum, adrenal gland, peritoneal region, sacrum). At this stage, palliative debulking surgery was performed on a painful subcutaneous lesion on the neck [29]. A section of tumor tissue was collected for the purpose of ex vivo therapy efficacy screening and a section was prepared for histopathology confirming metastatic urachal adenocarcinoma showing poorly differentiated neoplastic cells (Fig. 1b). Using standard techniques to establish cell cultures from human tissues [30], a primary cell culture was prepared for the drug screening experiment on the day of the surgery. Cytotoxicity of 1160 drug compounds representing all different FDA approved drug classes, with a fixed dose range of $1.25 \mu \mathrm{M}$ to $5 \mu \mathrm{M}$ was performed with the primary tumor cell culture (Mendeley data: DOI: https://doi.org/10.17632/ kc7wmn3rcs.2). With comparison of the negative control samples and hydroxyurea treated proliferation control samples, the estimated cell doubling rate of the primary culture was calculated to be $240 \mathrm{~h}$ corresponding to 0.3 cell division over the course of the 72 -h assay. To identify the most potent growth inhibitory drugs, effects of the drugs were averaged across all the test concentrations to derive a stringent ranking criterion where the growth inhibitory impact of the drug had to be stronger than the cell growth stalling effect of hydroxyurea (GR < 0 ) across all test doses (Fig. 2a). From this, 49 drugs resulting in a strong cytotoxic effect were nominated (Fig. 2b). These included 19 drugs used or developed for anticancer purposes (antimetabolites, microtubule poisons, nucleoside analogs, topoisomerase inhibitors, targeted therapeutics including WEE1, CDK4/6, mTOR, HDAC and a novel BET inhibitor ODM-207), as well as 30 drugs with other indications including statins and antibiotic compounds (Fig. 2b). The most potent growth inhibitory compound from the panel of 1160 drugs was Monensin, a monocarboxylic acid ionophore veterinary drug produced by Streptomyces cinnamonensis with antibiotic and anticancer activity [31,32].

\section{Validation using different ex vivo screening techniques}

To validate findings from the primary drug screen and to evaluate dependency of the drug efficacy profiles on the used assay technique, a repeated analysis of $90 \mathrm{se}-$ lected drugs with an expanded dose range was performed using three different high-throughput screening approaches; an enzymatic $2 \mathrm{D}$ cell viability assay, an imaging-based 2D cell viability assay and an image-based cell viability assay of cells cultured in 3D cell culture conditions (Fig. 3a). All drugs were tested with five 2fold concentrations and dose responses were normalized using GR metrics to correct for the differential measured cell growth rate of $\sim 0.6, \sim 0.5$ and $\sim 0.3$ cell doublings per $72 \mathrm{~h}$ in the image-based 2D, enzymatic $2 \mathrm{D}$ and image-based 3D assay respectively (Fig. 3b). Unsupervised hierarchical clustering of the dose responses across all three different assay methods was performed to identify patterns of response among the drug classes and to visualize variation in the response dependent on the used assay method (Fig. 3a). The overall most cytotoxic drugs independent of the assay method, based on growth rate normalized dose responses, were afatinib (2nd generation EGFRi, IC50 3,83 $\mu \mathrm{M}$ ), AZD2014 (mTOR1/2i, IC50 $0,36 \mu \mathrm{M}$ ), bortezomib (proteasome inhibitor, IC50 $1,24 \mu \mathrm{M}$ ), cladribine (purine analogue, IC50 3,57 $\mu \mathrm{M}$ ), ODM-207 (bromodomain inhibitor, IC50 1,01 $\mu \mathrm{M}$ ) and paclitaxel (taxane, IC50 3,38 $\mu \mathrm{M}$ ) (Fig. 3c, d). The median IC50 of mTOR inhibitor AZD2014 (vistusertib) when measured across the three different assay methods was lower by $\sim 10 \times$ to $\sim 20 \times$ in comparison to 5 -FU, 


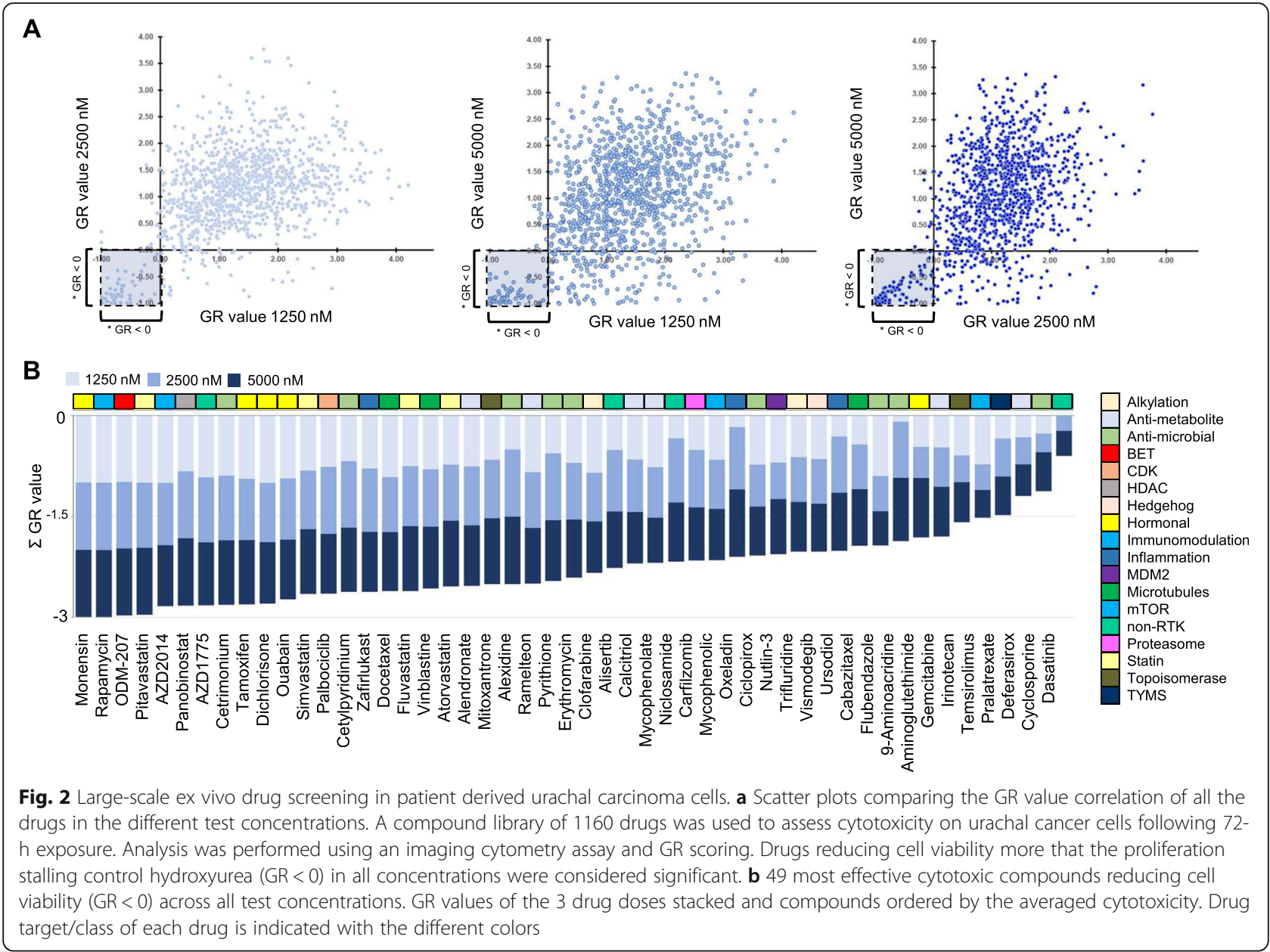

docetaxel, ciplatin or gemcitabine, agents that had been used to treat the patient. Interestingly, all included topoisomerase inhibitors; camptothecin, doxorubicin, irinotecan and topotecan resulted in more potent cytotoxic effects in the 3D cell culture assay (Fig. 3e) [33]. Overall, the drug response data measured with the enzymatic $2 \mathrm{D}$ assay and the image-based 2D assay had the highest degree of concordance across the full dose range (Pearson correlation, average across all doses, $r=0.45$ ). In general, the cytotoxic effects of majority of drugs were less potent in the 3D assay with the exception of topoisomerase inhibitors, docetaxel and vincristine.

\section{Evaluation of targeted drug combinations}

Results of the ex vivo screening indicated an apparent MAPK/PI3K signaling pathway switch in the tumor cells based on the high sensitivity of the cells to mTOR inhibition, MEK inhibition and partial response also to antifolate abitrexate, BRAF and EGFR inhibition. Other targeted therapeutics displaying cytotoxic effects were the ALK/ROS1/cMET inhibitor crizotinib, experimental bromodomain inhibitor ODM-207 and several VEGFR angiogenesis inhibitors (Fig. 3a). Efficacy of these compounds varied only little between the different ex vivo assay techniques indicating no dependency on the mode of cell growth (2D vs. 3D) (Fig. 3a). The RAS/RAF/ MEK/ERK and PI3K/AKT/mTOR signaling pathways all belong to mitogen-activated protein kinase (MAPK) signaling pathways. Mutations and/or activation by other mechanism of any one of the upstream genes (such as KRAS, BRAF, EGFR or MET) may result in abnormal activation of the signaling pathway converting into sensitivity towards inhibition of the MAPK signaling pathway [34]. Comparison of the sensitivity of patient derived tumor cells with the sensitivity of primary cultures previously derived from three urothelial bladder carcinomas and one small-cell neuroendocrine bladder carcinoma with no known MAPK pathway activating mutations [1] confirmed the selective sensitivity of the urachal cancer cells to the mTORC1/2 inhibitor AZD2014, MEK inhibitor trametinib and EFGR inhibitor afatinib (Supplementary Figure 2). To assess the genetic background of the patient's tumor cells a targeted oncopanel DNA sequencing was performed from tumor cells kept in continuous 

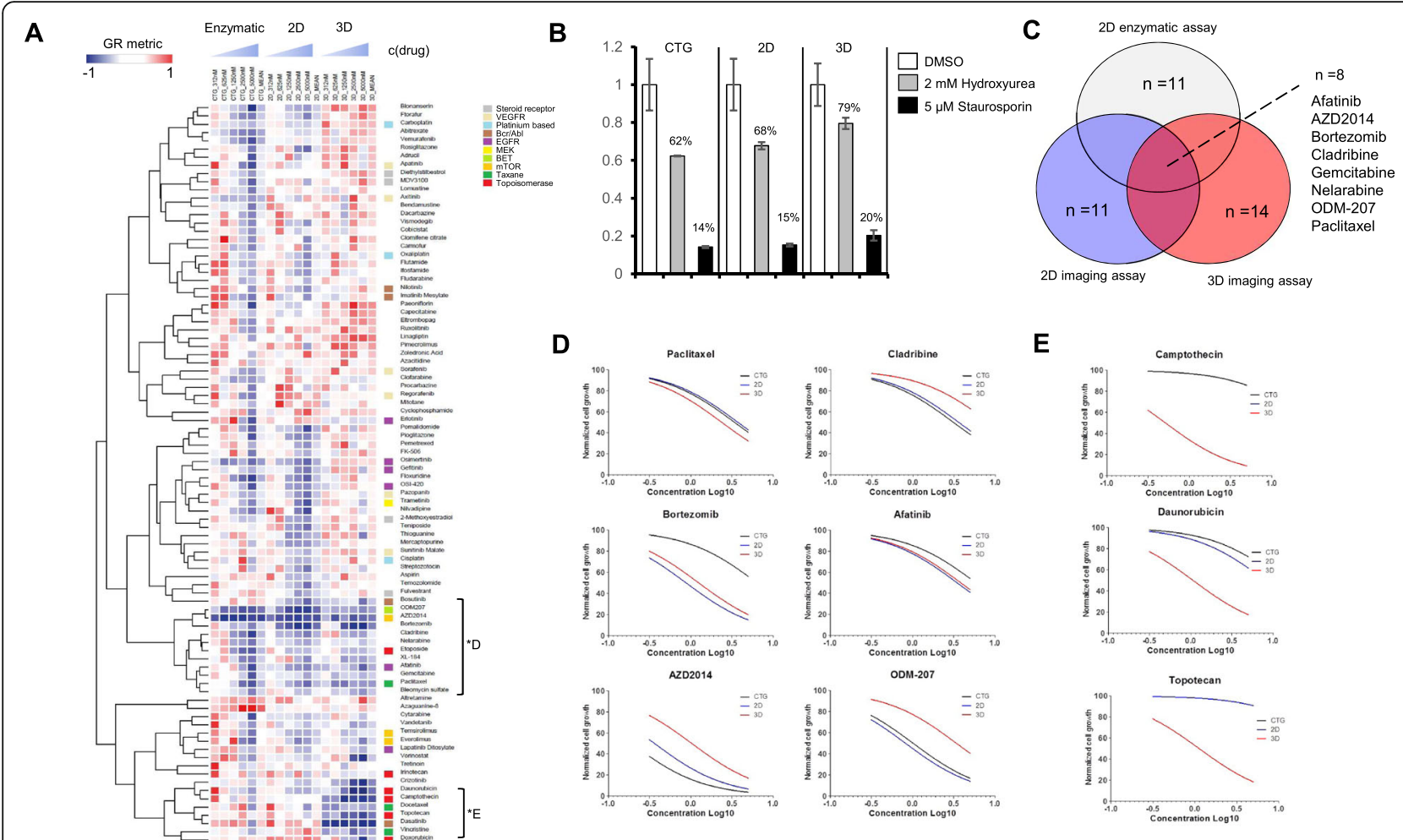

D

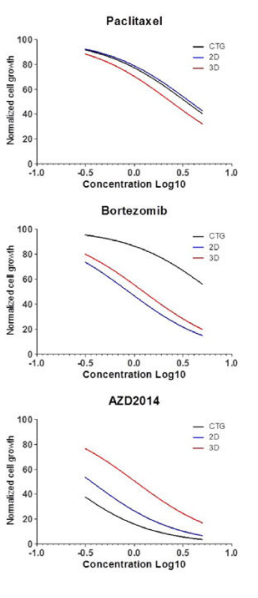

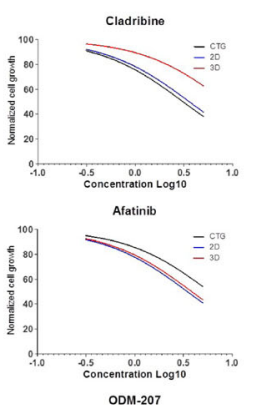

E

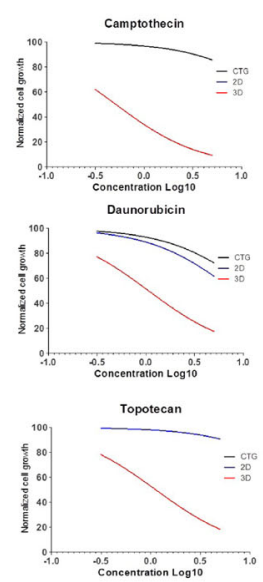

Fig. 3 Ex vivo validation screening using different assay techniques. a Heatmap display of vertical unsupervised clustering of the dose response data of the drugs from independent ex vivo screens using a 2D enzymatic cell viability assay, an image-based 2D cell viability assay and an image-based 3D cell viability assay. Each drug was tested in five concentrations. GR values $<0$ shown in blue. $\mathbf{b}$ Comparison of the assay controls used to calculate growth rates of the patient derived cells in the 2D and 3D culture conditions and measured with the different assay techniques. c Venn diagram showing the overlap of the top 30 most cytotoxic drugs from the different assay techniques. $\mathbf{d}$ Curve fitted dose response curves of the most potent cytotoxic drugs across all three different replicate screens. e Topoisomerase inhibitors had systematically a more potent cytotoxic effect in the 3D cell culture model assay

culture for 1 month following the drug screening experiments. Two known pathogenic mutations associated also with urachal cancers; FGFR4 and KRAS [23] and seven known mutations associated with a drug response were identified in the patient's tumor cells (Table 1.).

As suggested by the responsiveness of the patient's cells to MEK inhibitor trametinib [34], the tumor cells were found to harbor a somatic activating KRAS ${ }^{\mathrm{G} 12 \mathrm{~V}}$ mutation reflecting results from earlier studies reporting KRAS mutations being more common in UrAC than in urothelial bladder cancers [12]. For an in-depth view on potential drug combinations that could be synergistic with MEK inhibition in the treatment of KRAS mutated UrAC, we explored the effects of combining MEK inhibitor trametinib with the mTORC1/2 inhibitor AZD2014, ALK/cMET inhibitor crizotinib and BRAF

Table 1 Clinical significance SNVs identified in the patient's tumor cells

\begin{tabular}{|c|c|c|c|c|c|}
\hline Gene & AA Change & Codon Change & Mutation freq. & ClinVar ID & ClinVar significance \\
\hline$\overline{\mathrm{ABCB} 1}$ & p.S829A, pS893A & c.2677 T > G, c.2485 T > G & $31,8 \%$ & rs166622 & drug response \\
\hline DPYD & p.M166V & $c .495 A>G$ & $47,1 \%$ & rs100116 & drug response \\
\hline FGFR4 & p.G23R, p.G388R & c. $67 \mathrm{G}>\mathrm{A}$, p.1162G $>\mathrm{A}$ & $100,0 \%$ & rs16326 & pathogenic \\
\hline KRAS & p.G12V & $c .35 G>T$ & $71,6 \%$ & rs12583 & pathogenic \\
\hline SLCO1B1 & p.V174A & $\mathrm{C} .521 \mathrm{~T}>\mathrm{C}$ & $99,8 \%$ & rs37346 & drug response \\
\hline TAS2R38 & p.1296V & $c .886 \mathrm{~A}>\mathrm{G}$ & $99,9 \%$ & rs2906 & drug response \\
\hline TAS2R38 & p.A49P & c. $145 G>C$ & $100,0 \%$ & rs2904 & drug response \\
\hline TP53 & p.P33R, p.P72R & c. $.98 \mathrm{C}>\mathrm{G}, \mathrm{c} .215 \mathrm{C}>\mathrm{G}$ & $99,7 \%$ & rs12351 & drug response \\
\hline XPC & p.Q939K & c. $2815 \mathrm{C}>\mathrm{A}$ & $100,0 \%$ & rs190215 & drug response \\
\hline
\end{tabular}


inhibitor vemurafenib having cytotoxic effects both on the $2 \mathrm{D}$ and $3 \mathrm{D}$ assays (Supplementary Figure 1). The MISB18 cell line established from the patient's tumors were tested with a matrix of the inhibitor combinations in six doses. Trametinib was administered in six 3-fold dilutions starting from $2 \mu \mathrm{M}$ and the other drugs in six 3 -fold dilutions starting from $5 \mu \mathrm{M}$. Combination treatments with MEK and mTOR inhibition and MEK and ALK/cMET inhibition revealed additive effects of cytotoxicity (CI50 $=0.16$, CI50 $=0.45$ respectively), resulting in significant net reduction in cell numbers following 72 $\mathrm{h}$ of treatment (Fig. 4a). Cytotoxic IC50 of trametinib and AZD2014 as a combination at a fixed molar ratio of 1 to 2.5 was $<10 \mathrm{nM}$, compared to IC50 values of $80 \mathrm{nM}$ and $240 \mathrm{nM}$ of the drugs as single agents, respectively (Fig. 4a \& b). This finding is consistent with the role of kinome reprogramming and the alternate RTK signaling pathways feeding in the development of acute resistance to MEK inhibition cancer cells [35]. Also, the synergistic effect of inhibition of ALK/ROS1/cMET upstream of the MAPK pathway in combination with MEK inhibition fits concept as ROS1 inhibition has been previously shown to potentiate the anticancer effect of trametinib [35].

\section{Discussion}

The last decade of cancer research marks the era for evolution of the concept of personalized cancer care through the revolution of genetics and targeted therapies. In clinical oncology settings, the application of genomic medicine has been pioneered towards clinical trials where systemic cancer treatment is being targeted to individual patients based on molecular characterization of the patient's tumor [36]. This is particularly promising in context of clinical care of rare cancers, for which large clinical studies are not possible due to low number of cases. Treatment of rare cancers is often based on empirical approaches with standard chemotherapies. With advanced disease these treatments often fail, and no additional therapeutic options are available due to lack of clinical evidence on targeted treatments. In the future, combination of diagnostic therapy efficacy screening with genomic information [1$3,37-40$ ] could provide a robust diagnostic approach for personalized cancer medicine including immunooncology therapies [41] and thereby shift the clinical practice paradigm also in rare cancers. Currently the ex vivo screening techniques are under intense development and multiple different approaches has been described by different research groups [1-3, 37-41]. A common topic of debate regarding the ex vivo drug screening methods is the in vivo representativeness of the used models. 3D cell culture models have been promoted to increase the success rate of primary tumor cell cultures and resemble the primary tumors better than traditional two-dimensional (2D) cell culture models. This is in part due to potential transformation and loss

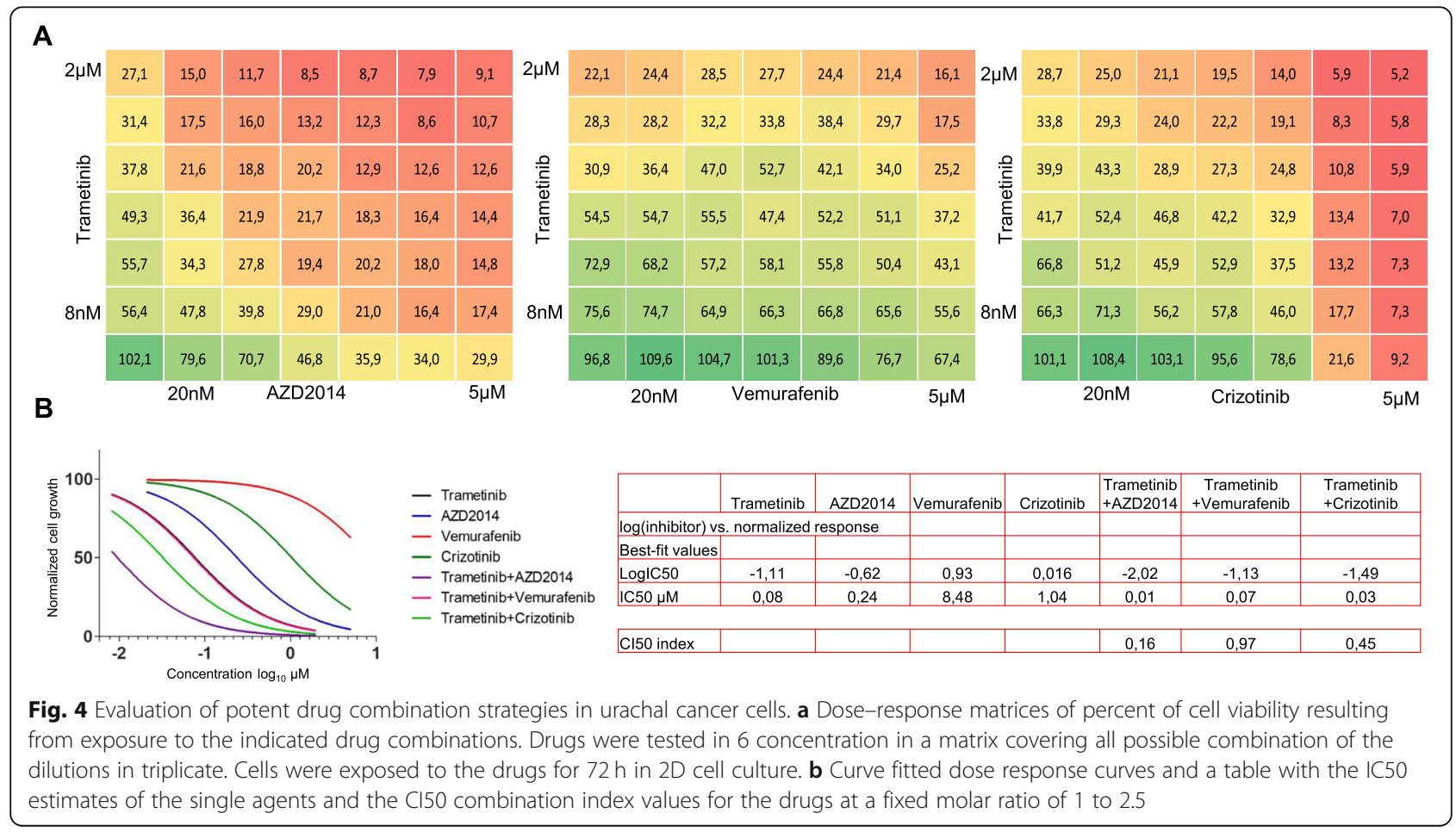


of heterogeneity of cancer cell cultures under conventional $2 \mathrm{D}$ in vitro propagation. Here, both $2 \mathrm{D}$ and $3 \mathrm{D}$ cell culture conditions were tested in parallel to compare the reproducibility of the methods. While the imagebased and enzymatic 2D cell culture assays had the best overall correlation of the dose response results, all three techniques yielded dose and target dependent cytotoxic profiles for drugs which could be linked directly to genomic features of the patient's cancer. Many drugs had also a significantly stronger cytotoxic effect in both the $2 \mathrm{D}$ and $3 \mathrm{D}$ assay than the standard chemotherapeutics that are being used for treatment of UrAC including the current patient. This shows that ex vivo therapy efficacy screening could be used as a rapid technique to complement pathological and clinical diagnostics to inform on treatment decisions [3]. Indeed, results from the first reported clinical trial utilizing ex vivo drug screening indicated a $88 \%$ overall response rate (ORR) for patients treated on basis of the approach [37]. This suggest that ex vivo screening has the potential for high accuracy in predicting responsive patients.

\section{Conclusion}

To evaluate feasibility and reproducibility of different ex vivo drug screening approaches to model therapeutic options for metastatic urachal adenocarcinoma, we performed a large-scale ex vivo drug screening using tumor cells freshly isolated from an UrAC tumor biopsy. The primary drug screening of 1160 drugs was initiated on the day of surgery and the screening results were available 4 days after sampling of the tissue. Findings from the initial screening were confirmed by alternative ex vivo screening techniques based on 2D and 3D cell culture models and two different assay approaches. All different assay techniques suggested sensitivity of the patient's tumor cells towards inhibition of MAPK signaling pathway targets MEK and mTOR. Targeted NGS profiling of the patient's cells confirmed an activating KRAS ${ }^{\mathrm{G} 12 \mathrm{~V}}$ mutation giving a rationale for the increased sensitivity of the tumor cells to MAPK signaling pathway. While our study is limited by the analysis of only a single UrAC patient sample, the significance of the results is the demonstration that rapid ex vivo screening without prior in vitro propagation of the patient derived tumor cells, both with $2 \mathrm{D}$ and $3 \mathrm{D}$ cell culture systems, identified drug sensitivities that reflected the genomic profile of the patient's tumor. Moreover, we describe the first UrAC cell line (MISB18) with a known tumor genomic profile, which together with future analyses of additional ex vivo UrAC samples can be used as a model to establish the role for pathogenic KRAS mutations on UrAC pathophysiology and drug sensitivity.

\section{Supplementary information}

Supplementary information accompanies this paper at https://doi.org/10. 1186/s12885-020-07092-w.

Additional file 1: Figure S1. Microscopic imaging of 2D and 3D urachal cancer cell cultures. Example transmitted light microscopy images of the phenotypes for the cytotoxic drugs at $1250 \mathrm{nM}$ concentration identified as potential therapeutics for urachal cancer cells based on the $2 \mathrm{D}$ and $3 \mathrm{D}$ ex vivo drug screening. Both brightfield microscopy and fluorescence microscopy imaging with DNA counterstaining with Hoechst (3D cultures) and DAPI (2D cultures) was performed with a 10x objective on an Olympus scan^R high content imager, bars $100 \mu \mathrm{m}$.

Additional file 2: Figure S2. Comparison of the drug primary urachal cancer cell culture to patient derived bladder cancer cell cultures. A) Heatmap visualization of the dose response of the urachal cancer cells MISB18 with four cell cultures established from patients samples of different bladder cancer types. GR values of $<0$ shown in blue. B) GR metrics describing the sensitivity of the cells to three drugs; afatinib, AZD2014 and trametinib displaying strongest selective cytotoxic effects on the urachal cancer cells in comparison to the bladder cancer cell cultures (data from image-based screening assays). Data available at Mendeley Data; DOI: https://doi.org/10.17632/kc7wmn3rcs.2.

\section{Abbreviations}

2D: 2 dimensional; 3D: 3 dimensional; ALK: Anaplastic lymphoma kinase; EGFR: Epidermal growth factor receptor; FGFR4: Fibroblast growth factor receptor 4; KRAS: KRAS proto-oncogene; mTOR: mammalian target of rapamycin; MAPK/MEK: Mitogen-activated protein kinase; NGS: Nextgeneration sequencing; UrAC: Urachal adenocarcinoma

\section{Acknowledgements}

We would like to thank the patient and the parents with whom this study is connected, Dr. Vesa Hongisto (Misvik Biology, Turku, Finland) for insights on $3 \mathrm{D}$ high-throughput drug screening experiments and the personnel of the Central Finland Health Care District, Jyväskylä Medical Center oncology care department. The ODM-207 bromodomain inhibitor was provided to the study kindly by Dr. Anu Moilanen (Orion Pharma, Turku, Finland).

\section{Authors' contributions}

Clinical materials and pathology: TK, JK. Conception and design: RM, JK, JKR. Development of methodology: RM, AA, VH, LP, JKR. Acquisition of data: RM, $A A, V H, N R, K R, J K R$. Analysis and interpretation of data: RM, VH, KR, TK, TP, JK, JKR. Writing, review and/or revision of the manuscript: RM, AA, VH, LP, KR, JK, JKR. Administrative, technical, or material support: TK, JK, TP, JKR. Study supervision: JKR. All authors read and approved the final manuscript.

\section{Funding}

This work has been supported in part by AZ-SLL-KI open innovation grant $\# 18122013$ without any influence in the design of the study and collection, analysis, and interpretation of data and in writing the manuscript.

\section{Availability of data and materials}

All data generated or analyzed during this study are included in this published article [and its supplementary information files]. Supplementary data is deposited to Mendeley data; DOl: https://doi.org/10.17632/

kc7wmn3rcs.2. The patient derived urachal cancer cell line MISB18 has been kept in continuous culture for an excess of 80 passages and is available from the corresponding author on reasonable request for non-profit research purposes in accordance to a specified MTA.

\section{Ethics approval and consent to participate}

The study was approved by the Institutional Review Board of the Central Finland Health Care District. The use and investigation of the patient derived cells was conducted in accordance with approval by the local Ethics Committee of the Central Finland Health Care District (KSSHP 3 U/2015). All experiments were undertaken with the understanding and written informed consent of the patient. The study methodologies conformed to the standards set by the Declaration of Helsinki. 


\section{Consent for publication}

Not applicable.

\section{Competing interests}

The authors of this manuscript declare that they have no competing interests. Juha K. Rantala is the founder of Misvik Biology Oy.

\section{Author details}

${ }^{1}$ Misvik Biology Ltd, Karjakatu 35 B, Fl-20520 Turku, Finland. ${ }^{2}$ Brinter Ltd, Turku, Finland. ${ }^{3}$ University of Sheffield, Sheffield, UK. ${ }^{4}$ Central Finland Health Care District, Jyväskylä, Finland. ${ }^{5}$ UPM-Kymmene Corporation, Helsinki, Finland. ${ }^{6}$ Eurofins Genomics Europe Sequencing GmbH, Constance, Germany. ${ }^{7}$ Docrates Hospital, Helsinki, Finland.

\section{Received: 6 May 2019 Accepted: 19 June 2020}

Published online: 23 June 2020

\section{References}

1. Kettunen K, Boström PJ, Lamminen T, et al. Personalized drug sensitivity screening for bladder cancer using conditionally reprogrammed patientderived cells. Eur Urol. 2019;76(4):430-4.

2. Lehtomaki Kl, Lahtinen LI, Rintanen N, et al. Clonal evolution of MEK/MAPK pathway activating mutations in a metastatic colorectal cancer case. Anticancer Res. 2019;39(11):5867-77.

3. Arjonen A, Mäkelä R, Härmä V, et al. Image-based ex vivo drug screen to assess targeted therapies in recurrent thymoma. Lung Cancer. 2020;145:27-32.

4. Billingham L, Malottki K, Steven N. Research methods to change clinical practice for patients with rare cancers. Lancet Oncol. 2016;17:e70-80.

5. Pauli $C$, Hopkins BD, Prandi $D$, et al. Personalized in vitro and in vivo cancer models to guide precision medicine. Cancer Discov. 2017;7(5):462-77.

6. Paner GP, Lopez-Beltran A, Sirohi D, Amin MB. Updates in the pathologic diagnosis and classification of epithelial neoplasms of urachal origin. Adv Anat Pathol. 2016;23(2):71-83.

7. Gopalan A, Sharp DS, Fine SW, et al. Urachal carcinoma: a clinicopathologic analysis of 24 cases with outcome correlation. Am J Surg Pathol. 2009;33: 659-68.

8. Ashley RA, Inman BA, Sebo TJ, Leibovich BC, Blute ML, Kwon ED, et al. Urachal carcinoma: clinicopathologic features and long-term outcomes of an aggressive malignancy. Cancer. 2006;107(4):712-20.

9. Yanagihara Y, Tanji N, Miura N, et al. Modified FOLFOX6 chemotherapy in patients with metastatic urachal cancer. Chemotherapy. 2013;59:402-6.

10. Mylonas KS, Malley PO, Ziogas IA, El-Kabab L, Nasioudis D. Malignant urachal neoplasms: a population-based study and systematic review of literature. Urol Oncol. 2017;35(1):33.e11-9.

11. Nagumo $Y$, Kojima $T$, Shiga $M$, et al. Clinicopathological features of malignant urachal tumor: a hospital-based cancer registry data in Japan. Int J Urol. 2020;2:157-62.

12. Hamilou Z, North S, Canil C, et al. Management of urachal cancer: a consensus statement by the Canadian Urological Association and genitourinary medical oncologists of Canada. Can Urol Assoc J. 2020;3:E57-64.

13. Szarvas T, Módos O, Niedworok C, Reis H, Szendröi A, Szász MA, et al. Clinical, prognostic, and therapeutic aspects of urachal carcinoma-a comprehensive review with meta-analysis of 1,010 cases. Urol Oncol. 2016; 34(9):388-98.

14. Kanamaru T, Iguchi T, Yukimatsu N, Shimizu Y, Kohyama Y, Tachibana H, et al. A case of metastatic urachal carcinoma treated with FOLFIRI (irinotecan and 5-fluorouracil/leucovorin) plus bevacizumab. Urol Case Rep. 2015;3(2):9-11.

15. Tran B, McKendrick J. Metastatic urachal cancer responding to FOLFOX chemotherapy. Can J Urol. 2010;17(2):5120-3.

16. Yazawa S, Kikuchi E, Takeda T, et al. Surgical and chemotherapeutic options for urachal carcinoma: report of ten cases and literature review. Urol Int 2012;88(2):209-14.

17. Hong SH, Kim JC, Hwang TK. Laparoscopic partial cystectomy with en bloc resection of the urachus for urachal adenocarcinoma. Int J Urol. 2007;14: 963-5.

18. Mertens LS, Behrendt MA, Mehta AM, et al. Long-term survival after cytoreductive surgery and hyperthermicintraperitoneal chemotherapy (HIPEC) for patients with peritonealmetastases of urachal cancer. Eur J Surg Oncol. 2019;45:1740-4.
19. Siefker-Radtke AO, Gee J, Shen Y, et al. Multimodality management of urachal carcinoma: the M. D. Anderson cancer center experience. J Urol. 2003;169:1295-8.

20. Molina JR, Quevedo JF, Furth AF, Richardson RL, Zincke $H$, Burch PA. Predictors of survival from urachal cancer: a Mayo Clinic study of 49 cases. Cancer. 2007;110(11):2434-40.

21. Sirintrapun SJ, Ward M, Woo J, et al. High-stage urachal adenocarcinoma can be associated with microsatellite instability and KRAS mutations. Hum Pathol. 2014;45:327-30.

22. Collazo-Lorduy A, Castillo-Martin M, Wang L, et al. Urachal carcinoma shares genomic alterations with colorectal carcinoma and may respond to epidermal growth factor inhibition. Eur Urol. 2016;70(5):771-5.

23. Reis $\mathrm{H}$, van der Vos KE, Niedworok C, et al. Pathogenic and targetable genetic alterations in 70 urachal adenocarcinomas. Int J Cancer. 2018;143(7): 1764-73.

24. Loh KP, Mondo E, Hansen EA, et al. Targeted therapy based on tumor genomic analyses in metastatic urachal carcinoma. Clin Genitourin Cancer. 2016;14(4):e449-52.

25. Doll S, Kriegmair MC, Santos A, et al. Rapid proteomic analysis for solid tumors reveals LSD1 as a drug target in an end-stage cancer patient. Mol Oncol. 2018;12(8):1296-307.

26. Módos $\mathrm{O}$, Reis $\mathrm{H}$, Niedworok $\mathrm{C}$, et al. Mutations of KRAS, NRAS, BRAF, EGFR, and PIK3CA genes in urachal carcinoma: Occurence and prognostic significance. Oncotarget. 2016;7(26):39293-301.

27. Landrum MJ, Lee JM, Benson M, et al. ClinVar: improving access to variant interpretations and supporting evidence. Nucleic Acids Res. 2018:46(D1): D1062-7.

28. Chou TC. Drug combination studies and their synergy quantification using the Chou-Talalay method. Cancer Res. 2010;70:440-6.

29. Sahu KK, Pandey D, Mishra AK, et al. Mystery of neck lump: an uncommon presentation of urachal cancer. BMJ Case Rep. 2019;12:e230215.

30. Labarge MA, Garbe JC, Stampfer MR. Processing of human reduction mammoplasty and mastectomy tissues for cell culture. J Vis Exp. 2013;71: 50011.

31. Westley JW, Evans RH Jr, Sello LH, Troupe N, Liu C, Miller PA. Isolation of novel antibiotics X 14667A and X-14667B from Streptomyces cinnamonensis subsp. urethanofaciens and their characterization as 2-phenethylurethanes of monensins B and a. J Antibiot (Tokyo). 1981;34:1248-52.

32. Ketola K, Vainio P, Fey V, Kallioniemi O, Iljin K. Monensin is a potent inducer of oxidative stress and inhibitor of androgen signaling leading to apoptosis in prostate cancer cells. Mol Cancer Ther. 2010;9(12):3175-85.

33. Niepel M, Hafner M, Mills CE, et al. A multi-center study on the reproducibility of drug-response assays in mammalian cell lines. Cell Syst. 2019:9(1):35-48.e5

34. Degirmenci U, Wang M, Hu J. Targeting aberrant RAS/RAF/MEK/ERK signaling for cancer therapy. Cells. 2020;9:1.

35. Zawistowski JS, Bevill SM, Goulet DR, et al. Enhancer remodeling during adaptive bypass to MEK inhibition is attenuated by pharmacologic targeting of the P-TEFb complex. Cancer Discov. 2017;7(3):302-21.

36. Mullard A. NCI-MATCH trial pushes cancer umbrella trial paradigm. Nat Rev Drug Discov. 2015;14(8):513-5.

37. Snijder B, Vladimer Gl, Krall N, et al. Image-based ex-vivo drug screening for patients with aggressive haematological malignancies: interim results from a single-arm, open-label, pilot study. Lancet Haematol. 2017;4(12):e595-606.

38. Puca $L$, Bareja $R$, Prandi $D$, et al. Patient derived organoids to model rare prostate cancer phenotypes. Nat Commun. 2018;9(1):2404.

39. Jeppesen M, Hagel G, Glenthoj A, et al. Short-term spheroid culture of primary colorectal cancer cells as an in vitro model for personalizing cancer medicine. PLoS One. 2017;12(9):e0183074.

40. Brijwani N, Jain M, Dhandapani M, et al. Rationally co-targeting divergent pathways in KRAS wild-type colorectal cancers by CANscript technology reveals tumor dependence on notch and Erbb2. Sci Rep. 2017;7(1):1502.

41. Powley IR, Patel M, Miles G, et al. Patient-derived explants (PDEs) as a powerful preclinical platform for anti-cancer drug and biomarker discovery. Br J Cancer. 2020;122(6):735 Epub ahead of print.

\section{Publisher's Note}

Springer Nature remains neutral with regard to jurisdictional claims in published maps and institutional affiliations. 